\title{
Cardiovascular protection in females linked to estrogen-dependent inhibition of arterial stiffening and macrophage MMP12
}

\author{
Shu-lin Liu, ${ }^{1}$ Anamika Bajpai, ${ }^{2}$ Elizabeth A. Hawthorne, ${ }^{1,3}$ Yongho Bae, ${ }^{1}$ Paola Castagnino, ${ }^{1,3}$ \\ James Monslow, ${ }^{4}$ Ellen Puré, ${ }^{4}$ Kara L. Spiller, ${ }^{2}$ and Richard K. Assoian ${ }^{1,3}$ \\ 'Department of Systems Pharmacology and Translational Therapeutics, Perelman School of Medicine, University of \\ Pennsylvania, Philadelphia, Pennsylvania, USA. ${ }^{2}$ School of Biomedical Engineering, Science and Health Systems, Drexel \\ University, Philadelphia, Pennsylvania, USA. ${ }^{3}$ Center for Engineering MechanoBiology and ${ }^{4}$ Department of Biomedical \\ Sciences, University of Pennsylvania, Philadelphia, Pennsylvania, USA
}

\begin{abstract}
Arterial stiffening is a consequence of aging and a cholesterol-independent risk factor for cardiovascular disease (CVD). Arterial stiffening and CVD show a sex bias, with men more susceptible than premenopausal women. How arterial stiffness and sex interact at a molecular level to confer risk of CVD is not well understood. Here, we used the sexual dimorphism in LDLR-null mice to show that the protective effect of female sex on atherosclerosis is linked to reduced aortic stiffness and reduced expression of matrix metalloproteinase-12 (MMP12) by lesional macrophages. Deletion of MMP12 in LDLR-null mice attenuated the male sex bias for both arterial stiffness and atherosclerosis, and these effects occurred despite high serum cholesterol. Mechanistically, we found that oxidized LDL stimulates secretion of MMP12 in human as well as mouse macrophages. Estrogen antagonizes this effect by downregulating MMP12 expression. Our data support cholesterol-independent causal relationships between estrogen, oxidized LDL-induced secretion of macrophage MMP12, and arterial stiffness that protect against atherosclerosis in females and emphasize that reduced MMP12 functionality can confer atheroprotection to males.
\end{abstract}

Authorship note: SLL and $A B$ are co-first authors. KLS and RKA are co-corresponding authors.

Conflict of interest: The authors have declared that no conflict of interest exists.

License: Copyright 2019, American Society for Clinical Investigation.

Submitted: June 7, 2018

Accepted: November 20, 2018

Published: January 10, 2019

\section{Reference information:}

JCI Insight. 2019;4(1):e122742.

https://doi.org/10.1172/jici.

insight.122742.

\section{Introduction}

Atherosclerosis is a disease of aging and inflammation. Although often viewed in the context of aberrant cholesterol biology, the mechanisms responsible for atherosclerosis are multifaceted and complex and extend beyond dyslipidemia (1-5). A striking example comes from epidemiologic studies in humans, which have identified arterial stiffening as a cholesterol-independent risk factor for a first cardiovascular event (6-9). Indeed, recent work by us and others shows that increased ECM and arterial stiffness stimulate endothelial permeability, leukocyte transmigration, and monocyte/macrophage adhesion to subendothelial matrix proteins $(10,11)$. Conversely, pharmacologic blockade of arterial stiffening can reduce atherosclerotic lesion burden in apolipoprotein E-null (apoE-null) mice even in the presence of high cholesterol (10)

Arterial stiffness reflects the counterbalancing effects of fibrillar collagens and elastin. Fibrillar collagens contribute to the strain-stiffening property of arteries while elastin allows for recoil (12). Newly synthesized fibrillar collagens and elastin are also cross-linked by lysyl oxidases, and this cross-linking contributes to the mechanical properties of these proteins. Although fibrillar collagens can be synthesized and cross-linked throughout life, elastin biosynthesis is restricted to prenatal and early postnatal life (12-15). Thus, elastin-associated changes in arterial stiffness are mostly regulated by degradation. MMPs have important roles in regulating ECM protein degradation, and MMP inhibition has been tested clinically though outcomes have been confounded by the use of broad-spectrum inhibitors (16).

We previously described an important role for MMP12, a potent elastase $(17,18)$, in arterial stiffening with age and in the response to vascular injury in mice (19). Although the MMP12 effect on aging- and injury-associated arterial stiffening was attributed primarily to its expression by vascular smooth muscle cells (SMCs) (19), the role of MMP12 in animal models of atherosclerosis has more commonly been associated with its expression by macrophages (20-22). Additionally, work by Johnson et al. (23) suggests a 
potential therapeutic benefit of MMP12 inhibition because a highly specific MMP12 pharmacologic inhibitor attenuated atherosclerosis in apoE-null mice. The reduced lesion burden in these mice was associated with decreased elastin fragmentation and decreased macrophage abundance/invasion (23). The extrapolation of results from mice to humans must be viewed with caution, but MMP12 has also been identified as a candidate locus in human atherosclerosis, stroke, and cardiometabolic disease (24-27). In particular, MMP12 protein has been localized to macrophage-rich $\left(\mathrm{CD}^{+} 8^{+}\right)$regions of human atherosclerotic plaques, where its expression correlates with elastin breakdown (26). Yet how MMP12 promotes atherosclerosis and how its expression is regulated remains largely unknown despite the likely importance of these issues in helping to identify patient populations that might benefit from MMP12 inhibition.

The relationship between age and atherosclerosis is particularly evident in women, who are relatively protected from cardiovascular disease until menopause (28-34). The beneficial cardiovascular characteristics of female sex are diverse and include decreased blood pressure, a beneficial serum lipid profile, improved endothelial function, decreased abundance of thrombotic plaques, reduced oxidative stress, and increased expression of SMC Cox2 (28, 30, 35-37). Many, but not all, of these protective phenotypes have been linked to the antiatherogenic effects of estrogen acting through estrogen receptor- $\alpha$ (28, 30, 35, 37). Arterial stiffness is also reduced in premenopausal women as compared with age-matched men, and estrogen replacement therapy has been reported to decrease arterial stiffness in postmenopausal women (31-34). But how these sex and estrogen effects on arterial stiffness and atherosclerosis are linked causally or mechanistically is not known. Here we used the known sexual dimorphism of the LDLR-null mouse model $(37,38)$ to causally link reduced expression of MMP12 to reduced arterial stiffness and atherosclerosis in females. Mechanistically, we show that estrogen selectively inhibits MMP12 expression and secretion in macrophages treated with oxidized LDL (Mox macrophages). Remarkably, deletion of MMP12 in the LDLR-null mouse extends atheroprotection to males even in the presence of high cholesterol.

\section{Results}

Sex differences in atherosclerosis linked to arterial stiffness and MMP12. To examine relationships between female sex, arterial mechanics, and atherosclerosis, female $\mathrm{LDLR}^{-/-}$mice were bilaterally ovariectomized and then placed on a high fat-diet in the absence and presence of infused 17ß-estradiol (E2) from 8 to 24 weeks of age. Atherosclerotic lesion burden (Figure 1, A and B) in the female LDLR ${ }^{-1-}$ mice was increased upon ovariectomy (compare arrow and left box in Figure 1B). Estrogen replacement strongly reduced lesion burden in these ovariectomized mice (Figure 1, A and B). Atomic force microscopy (AFM) showed that these effects of ovariectomy and exogenous E2 on lesion burden were matched by changes in arterial stiffness (Figure 1C). Plasma cholesterol levels remained highly elevated in the ovariectomized mice with or without E2 replacement (Figure 1D). The data support a cholesterol-independent causal relationship between estrogen, atherosclerosis, and arterial stiffness in female mice.

We searched for potential molecular targets of the estrogen effect on arterial stiffening in atherosclerosis by comparing the gene expression profiles of several atherosclerosis-associated ECM components and ECM-regulating MMPs in the aortas of male $\mathrm{LDLR}^{-/-}$mice before and after high-fat diet (Supplemental Figure 1A; supplemental material available online with this article; https://doi.org/10.1172/jci. insight.122742DS1). We did not find pronounced differences in the gene expression profiles of collagen type I (the most abundant arterial fibrillar collagen), fibronectin, or lysyl oxidase in $\mathrm{LDLR}^{-/}$mice with time on a high-fat diet. However, we did find a striking induction of MMP12 mRNA with time on a high-fat diet that greatly exceeded the differential expression of MMP2 or MMP9, 2 MMPs frequently studied in the context of vessel wall remodeling and atherosclerosis $(22,39,40)$. These results support prior work showing that MMP12 is highly expressed in atherogenic lesions and that transgenic MMP12 expression stimulates atherosclerosis while MMP12 depletion or pharmacologic inhibition reduces atherosclerosis in animal models (20, $21,23,41,42)$. Moreover, interrogation of an existing genome-wide analysis of aortas from apoE ${ }^{-/-}$mice (GSE13865) showed that the levels of MMP12 mRNA greatly exceeded that of any other MMP, particularly in the atheroprone regions (Supplemental Figure 1B). Thus, atherosclerosis in the 2 most commonly used mouse models is associated with a pronounced and preferential increase in MMP12 gene expression.

Because MMP12 can degrade elastin and increase arterial stiffness (19), we hypothesized that MMP12 expression might be lower in female $\mathrm{LDLR}^{-/-}$mice than age-matched males. Indeed, male $\mathrm{LDLR}^{-/-}$mice expressed more MMP12 protein in macrophage-containing $\left(\mathrm{CD}^{+}\right)$aortic root lesions than the $\mathrm{LDLR}^{-/}$ females (Figure 1, E and F, closed arrowheads, and Supplemental Figure 2). In contrast, we did not notice 
A

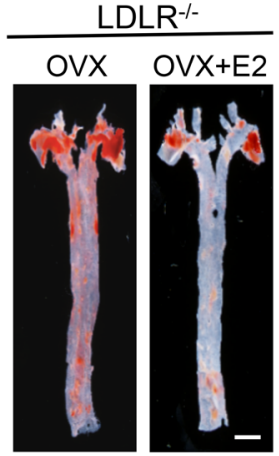

C

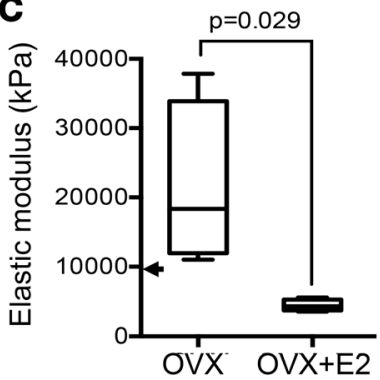

$\mathbf{E}$
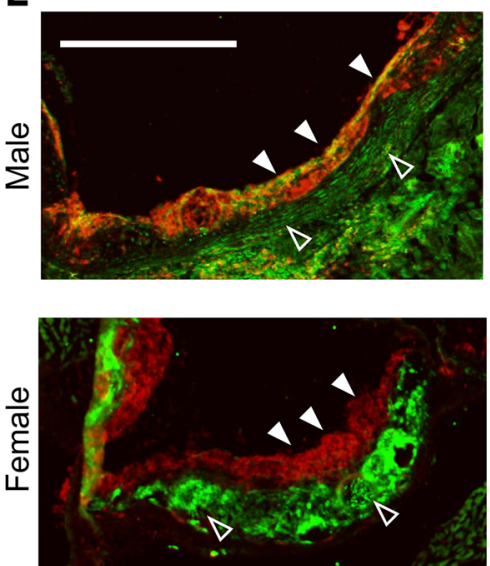

CD68 MMP12
B
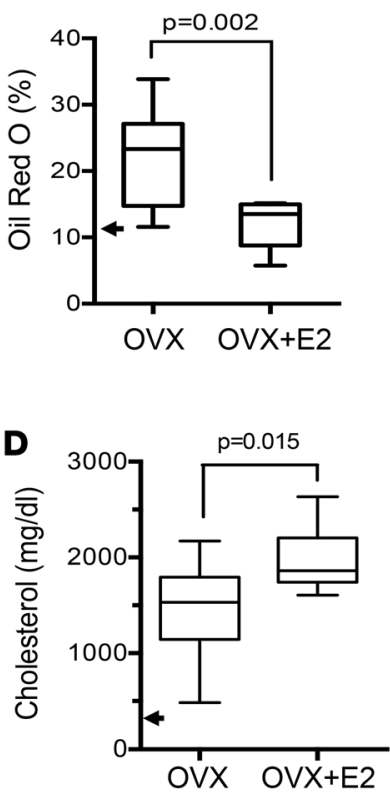

$\mathbf{F}$



Figure 1. Protective effects of estrogen and female sex on arterial stiffness and atherosclerosis linked to reduced expression of MMP12. (A) Representative images of atherosclerotic lesion burden of ovariectomized (OVX) $\mathrm{LDLR}^{-/-}$ mice fed a high-fat diet from 8 to 24 weeks of age, with or without exogenous estradiol (OVX+E2). Lipid-rich lesions were identified by en face Oil Red $\mathrm{O}$ staining in aortas from OVX $(n=10)$ and OVX+E2 groups $(n=12)$. Scale bar: $1 \mathrm{~mm}$. (B) Quantification of data from A expressed as a percentage of aortic area. (C) Arterial stiffness (elastic modulus) determined by AFM; $n=4$ per group. The arrowheads in $B$ and $C$ represent the median Oil Red 0 staining and elastic moduli of 6-month female LDLR ${ }^{-/-}$mice on a high-fat diet without OVX (taken from Figure 2). (D) Blood cholesterol levels were measured after completion of the high-fat diet ( $n=10$ per condition). The arrow approximates the cholesterol level in C5BL/6 mice on a Western diet (71). (E) Aortic root sections of male and female LDLR ${ }^{-1-}$ mice on a high-fat diet from 8 to 24 weeks costained for CD68 (red) and MMP12 (green). The images were merged to show colocalization; see Supplemental Figure 2 for individual images. Closed and open arrowheads show MMP12 levels in CD68+ and CD68- regions, respectively. Scale bar: $500 \mu \mathrm{m}$. (F) Quantification of MMP12 signal intensity in $\mathrm{CD}^{2} 8^{+}$regions from $\mathbf{E}$ ( $n=5$ per group). Graphs show box and whisker plots with Tukey's whiskers; the horizontal lines of boxes represent the 25th percentile, the median, and the $75 \%$ percentile. Statistical significance for all panels was determined using Mann-Whitney tests.

a reduced expression of MMP12 in the (largely SMC-derived) stromal compartment of aortic root lesions from male versus female $\mathrm{LDLR}^{-/-}$mice (Figure 1, E and F, open arrowheads). Total collagen abundance, determined by trichrome staining, was similar in aortic root sections of male and female LDLR $^{-/-}$mice (Supplemental Figure 3). 
Because of the increasingly appreciated role of cellular senescence in the pathogenesis of atherosclerosis (43), we wondered if the reduced arterial stiffness and MMP12 expression seen in female arteries might be related to an effect of MMP12 on cell senescence. We compared arteries of WT and MMP12-null mice for expression of $\mathrm{p} 16^{\mathrm{INK} 4 \mathrm{a}}$, an established senescence marker. Consistent with other studies (44-46), we found both cytoplasmic and nuclear staining for p16 $6^{\mathrm{INK} 4 \mathrm{a}}$ (Supplemental Figure 4A), but the signal intensities were independent of MMP12 status (Supplemental Figure 4B). Similarly, MMP12 did not affect blood pressure in 6-month-old mice of either sex (Supplemental Figure 4C), a result also seen by others in atheroprone mice (47).

Male sex bias for arterial stiffening and atherosclerosis eliminated by deletion of MMP12. We generated male and female MMP12 $2^{-/-}$mice on the LDLR ${ }^{-/-}$background and placed them on a high-fat diet from 8 to 24 weeks to determine whether differential MMP12 expression could explain the effect of sex on arterial stiffness and lesion burden. In situ enzymatic activity assays indicated that MMP12 contributes about half of total lesional elastase activity (Supplemental Figure 5, A and B). The levels (Supplemental Figure 5, C and D) and structure (Supplemental Figure 5E) of collagen type I were similar in the presence or absence of MMP12.

Consistent with results in Figure 1, AFM revealed direct relationships between sex, arterial stiffness, and lesion burden in $\mathrm{LDLR}^{-1-}$ mice on a high-fat diet. The aortas of the males were stiffer than the females (Figure 2A), and the extent of Oil Red O staining was greater (Figure 2, B and C). Moreover, deletion of MMP12 eliminated the male sex bias in both arterial stiffness and atherosclerosis, reducing both parameters to the levels seen in females (Figure 2, A and C). The cardiovascular risk associated with increased arterial stiffness is cholesterol-independent in humans (6-9). Similarly, the sex-associated changes in arterial stiffness and lesion burden of $\mathrm{LDLR}^{-/-}$mice seen in response to female sex and deletion of MMP12 occurred without changes in total blood cholesterol and despite its elevation by high-fat diet (Figure 2D).

Cellular analysis of aortic root lesions showed that macrophage abundance, as determined by staining for CD68, was low in high-fat-fed female $\mathrm{LDLR}^{-/-}$mice as compared with age-matched males; this sex difference was also attenuated by deletion of MMP12 (Figure 3, A and B). In contrast, SMC content in these lesions, as determined by staining for $\alpha$-SMA, was less affected by sex or the presence or absence of MMP12 (Figure 3, A and C).

Selective inhibition of MMP12 expression by estrogen in human macrophages. We then directly tested the relationships between estrogen and MMP12 in macrophages and SMCs. To make the macrophage experiments particularly relevant to atherosclerosis, female blood-derived human monocytes were differentiated in vitro with macrophage colony stimulating factor (MCSF) and treated with oxidized LDL to generate the Mox phenotype (48). The Mox cells were then incubated with selected concentrations of E2 (Figure 4A). Interestingly, oxidized LDL increased MMP12 protein levels in the condition media of the human Mox macrophages compared with the untreated controls (M0) (Figure 4B), and this effect was independent of changes in MMP12 gene expression (Figure 4C). In contrast, E2 inhibited levels of secreted MMP12 in these human cells (Figure 4D), and this inhibitory effect corresponded to reduced expression of MMP12 mRNA (Figure 4E). In contrast, E2 had minimal effect on MMP12 gene expression in human female SMCs (Figure 4F). We repeated this entire analysis in bone marrow-derived mouse macrophages and found that the inhibitory effects of E2 on MMP12 gene expression and secretion were also observed in mouse Mox cells (Supplemental Figure 6, A-D), whereas MMP12 expression in female mouse SMCs was resistant to E2 (Supplemental Figure 6E).

As expected, Oil Red $\mathrm{O}$ staining was increased in Mox macrophages relative to the $\mathrm{M} 0$ controls (Figure 5, A and B). However, inhibition of MMP12 gene expression by E2 occurred without reduction in the level of Oil Red O staining (Figure 5, A and C), indicating that the estrogen-mediated inhibition of macrophage MMP12 is not due to efflux of oxLDL. Neither cell viability (Supplemental Figure 7A) nor cell senescence, as judged by the expression of $\mathrm{p} 16^{\mathrm{INK} 4 \mathrm{a}}$ (Supplemental Figure 7, B and C), were affected by E2.

\section{Discussion}

Our results indicate that inhibition of macrophage MMP12 expression contributes to the protective effect of female sex on arterial stiffness and atherosclerosis and that this effect is independent of changes in serum cholesterol. Mechanistically, we show that the uptake of oxLDL causes an increase in MMP12 secretion by macrophages, and this increase is mitigated by estrogen, thus pointing to a potential mechanism by which female sex protects from atherosclerosis. Because arterial stiffening increases macrophage adhesion to substratum (10), we suggest that the ability of estrogen to reduce lesional 
A

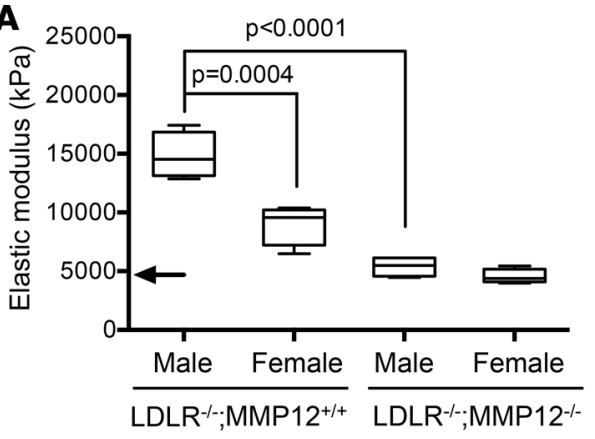

B

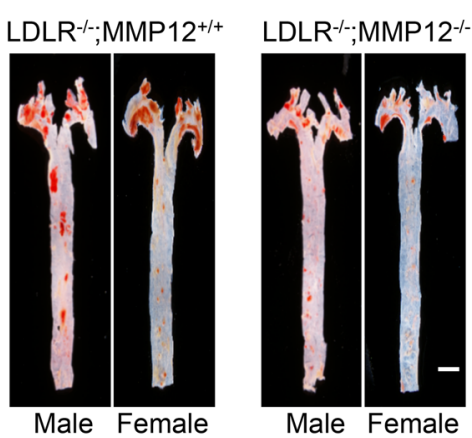

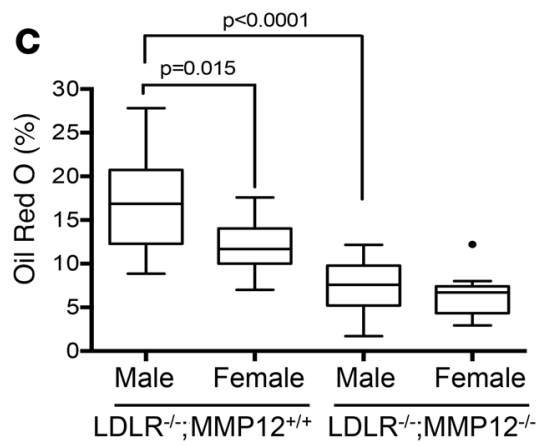

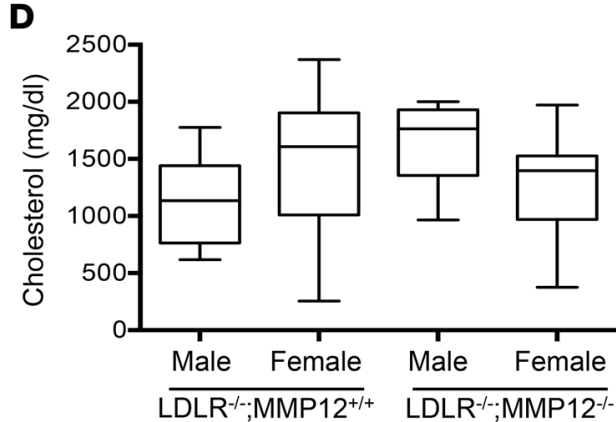

Figure 2. Deletion of MMP12 eliminates the male sex bias for arterial stiffening and atherosclerosis. Male and female $\mathrm{LDLR}^{-/-} \mathrm{MMP12}^{+/+}$and $\mathrm{LDLR}^{-/-} \mathrm{MMP12}^{-/-}$mice were fed a high-fat diet from 8 to 24 weeks before analysis. (A) Small portions of the descending aortas from the $\mathrm{LDLR}^{-/-}$and $\mathrm{MMP12}^{-{ }^{--}} \mathrm{LDLR}^{-/-}$mice were analyzed by AFM $(n=4$ for each group). The arrow indicates the median elastic modulus of a WT aorta (10). (B) Lipid-rich atherosclerotic lesions were identified by en face Oil Red 0 staining of isolated aortas from male $\operatorname{LDLR}^{-/-}(n=10)$ and $\operatorname{LDLR}^{-/-} \mathrm{MMP}^{-1-}(n=14)$ mice and female LDLR ${ }^{-/-}(n=10)$ and $\mathrm{LDLR}^{-/-} \mathrm{MMP12}^{-/-}(n=11)$ mice. Scale bar: $1 \mathrm{~mm}$. (C) Quantitation of results in B expressed as a percentage of the aortic area. The dot indicates a statistical outlier. (D) Blood cholesterol levels of male $\operatorname{LDLR}^{-1-}(n=$ 9) and $\mathrm{LDLR}^{-/-} \mathrm{MMP12}^{-/-}(n=14)$ mice and female $\mathrm{LDLR}^{-/-}(n=10)$ and $\mathrm{LDLR}^{-/-} \mathrm{MMP12}^{-/-}(n=12)$ mice were determined at the time of sacrifice. Graphs show box and whisker plots with Tukey's whiskers; the horizontal lines of boxes represent the 25th percentile, the median, and the $75 \%$ percentile. Statistical significance for all panels was determined by ANOVA.

MMP12 in females short-circuits a feedback loop that perpetuates arterial stiffening and lesional macrophage abundance (Figure 5D). We note that MMP12 is also expressed in lesional SMCs, and SMC MMP12 likely contributes to the overall pool of elastase activity in atherosclerosis. However, the similar levels of stromal MMP12 in male and female $\mathrm{LDLR}^{-/-}$mice and, in particular, the lack of an estrogen effect on MMP12 gene expression by cultured SMCs indicate that the sex dependency of arterial stiffening and atherosclerosis is more strongly linked to the MMP12 expressed in macrophages.

In addition to our work, which reveals a likely atheroprotective benefit of reduced arterial stiffness (this report and ref. 10), we note complementary findings by others demonstrating a direct relationship between increased arterial stiffness and increased atherosclerosis in atheroprone mice (49-52). Two recent reports using heterozygous expression of elastin indicate that increased arterial stiffness likely accelerates (rather than increases the maximal amount of) lesion burden and that this effect may be site specific and involve nonmechanical factors such as blood pressure and circulating soluble factors $(49,53)$.

Although several studies have implicated MMP12 in atherosclerotic lesion burden $(20-23,41,42)$, the direct effects of oxLDL on macrophage MMP12 have not been previously described, perhaps because of a lack of effects on the transcription level (this report and ref. 54). Similarly, others have not observed a sex difference in lesion burden between male and female high-fat-fed apoE-null mice given either vehicle or a pharmacologic MMP12 inhibitor (23). This difference in results may reflect subtleties of experimental conditions or the reduced sexual dimorphism of the apoE-null mouse as compared with LDLR-null mice (38). Our data in the LDLR-null mice suggest that MMP12 inhibition therapy might particularly benefit men with high cholesterol who are not responsive to standard treatment, such as statins. Direct testing of this model will be an important matter for further study. 
A
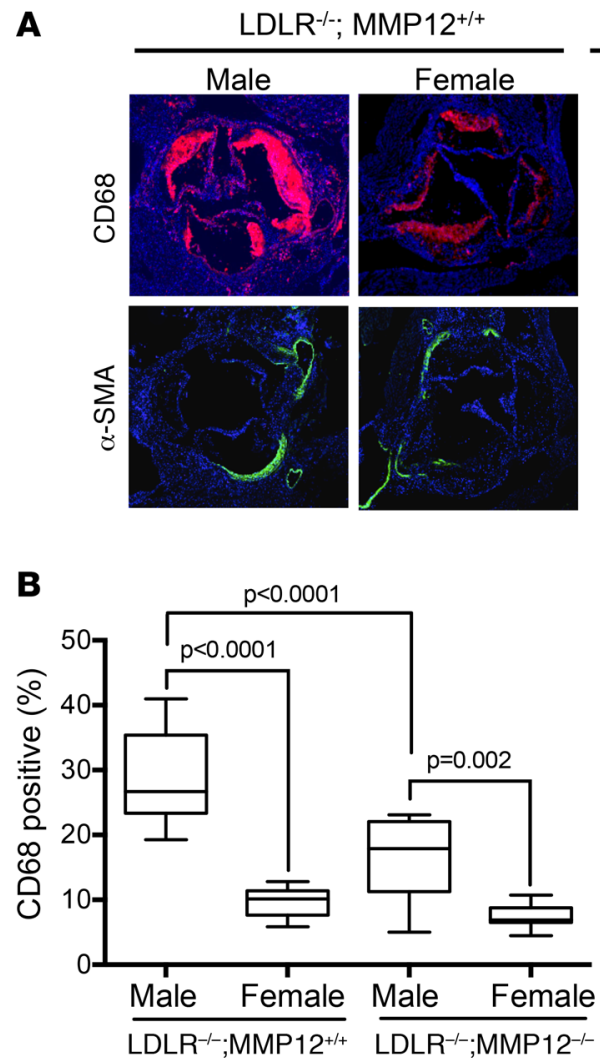

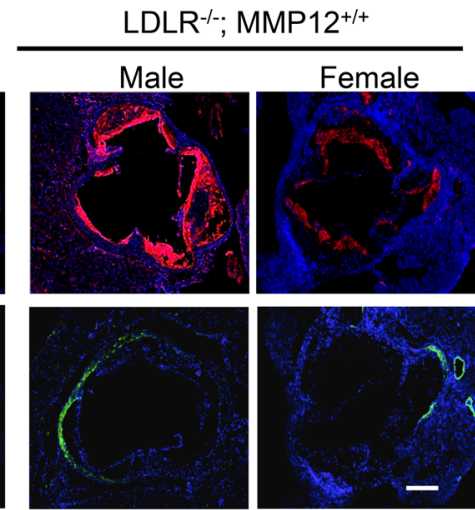

C

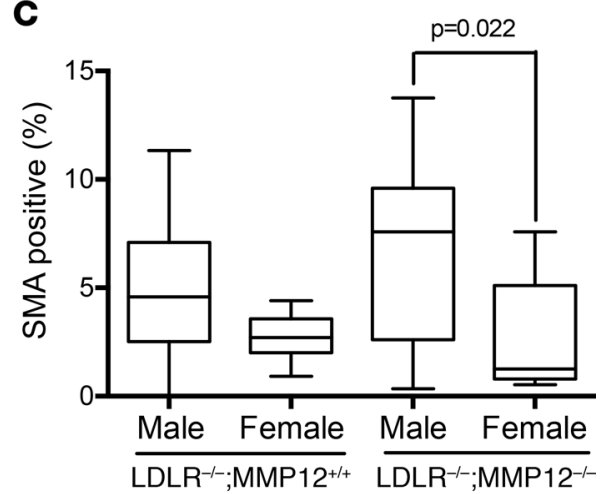

Figure 3. Reduced macrophage content in aortic root lesions of female LDLR-null mice. Male and female LDLR ${ }^{-/-}$ $\mathrm{MMP}^{+/+}$and $\mathrm{LDLR}^{-/-}$MMP12/- mice were fed a high-fat diet from 8 to 24 weeks before analysis of aortic root sections. (A) Aortic root cross-sections from male LDLR ${ }^{-/-}(n=10)$ and LDLR ${ }^{-/-} \mathrm{MMP12}^{-/-}(n=10)$ mice and female LDLR ${ }^{-/-}(n=$ 10) and LDLR ${ }^{-/-}$MMP12-/- $(n=9)$ mice stained with CD68 (red) and $\alpha$-smooth muscle actin ( $\alpha$-SMA) (green). Scale bar: $200 \mu \mathrm{m}$. (B and C) Quantification of results in A expressed as a percentage of the aortic root area. Graphs show box and whisker plots with Tukey's whiskers; the horizontal lines of boxes represent the 25th percentile, the median, and the 75\% percentile. Statistical significance for $\mathbf{B}$ and $\mathbf{C}$ was determined by ANOVA.

How might elastin degradation by MMP12 contribute to arterial stiffening? Fibrillar collagens and elastin have distinguishable and complementary roles in regulating arterial stiffness, with elastin bearing most of the mechanical load at relative low pressures and fibrillar collagen (primary collagen type I) bearing most of the load at higher pressures $(12,55)$. When isolated arteries are treated with elastase, adventitial collagen fibers straighten (56), an indicator of strain stiffening. These studies suggest that elastin degradation by MMP12 may lower the threshold at which the mechanical burden is shifted to strain-stiffening arterial collagens.

Although our results are consistent with female sex and estrogen reducing MMP12-associated elastase activity, we do not exclude the possibility that sex differences in MMP12 abundance may also affect arterial stiffness indirectly through effects on other MMPs, other ECM components, or matrikines (57-59). It is also possible that the reduced lesional abundance in $\mathrm{MMP} 12^{-/-}$mice results in part from non-stiffness-mediated effects of MMP12. For example, cell infiltration of MMP12 $2^{-/-}$macrophages and PMNs into basement membrane is reduced in vitro and in vivo (60-62). In addition, MMP12 can exert proinflammatory functions, including stimulating release of cytokines like TNF $\alpha$ and IL-6 $(63,64)$ as well as production of inducible nitric oxide synthase (65); its loss may reduce leukocyte recruitment, proliferation, survival, and function. New work suggests intracellular MMP12 has effects on transcription (66). Thus, inhibition of macrophage-derived MMP12 by estrogen may reduce lesional abundance and protect from inflammatory damage in multiple ways. Conversely, multiple biological pathways can affect arterial stiffness (67), and these pathways may also contribute to the protective effects of female sex and estrogen. Finally, the dose of estrogen may be important and should be thoroughly assessed in future studies, as well as potential contributory effects of estrogen from other cell types. 
A

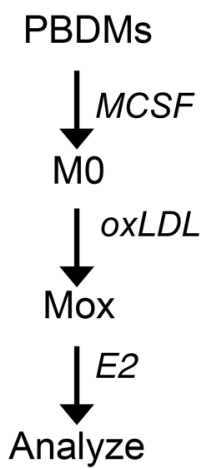

B



C

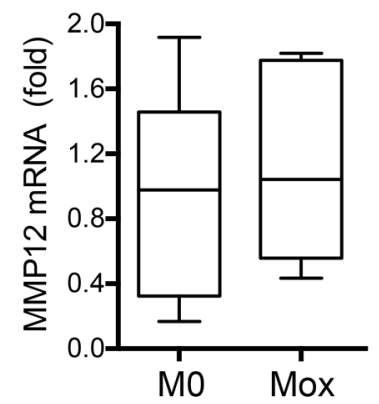

D
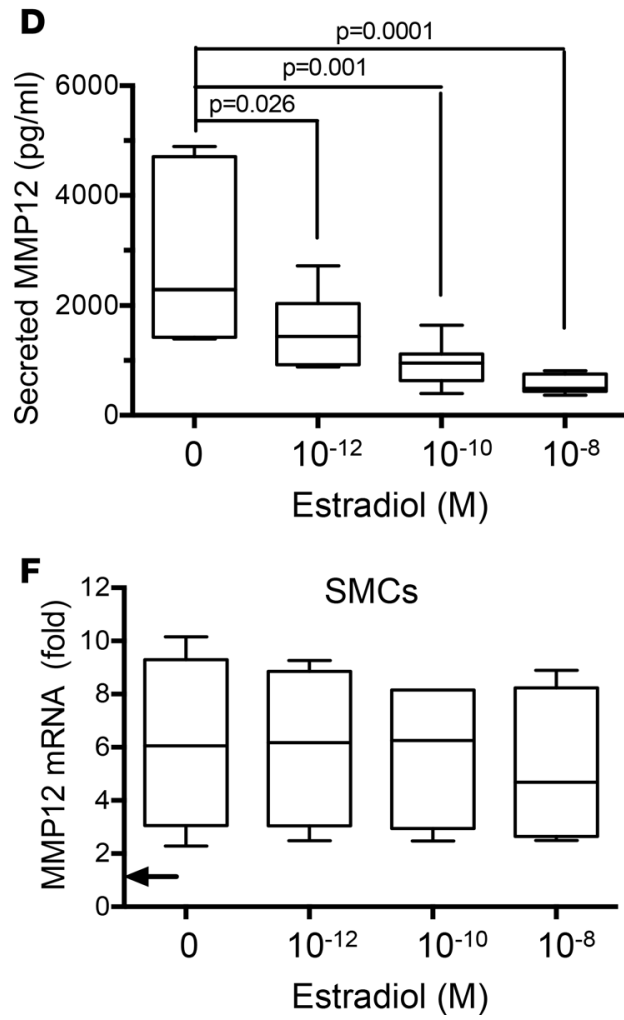

$\mathbf{E}$



Figure 4. Estrogen inhibits MMP12 gene expression and secreted MMP12 in human Mox macrophages. (A) Schematic of the experimental design. Female human peripheral blood-derived monocytes (PBDMs) were differentiated into MO macrophages, treated with oxidized LDL (oxLDL), and exposed to selected concentrations of E2 as described in Methods. (B and C) Effect of oxLDL on the amount of secreted MMP12 and MMP12 mRNA, respectively, in MO versus Mox macrophages ( $n=7$ per condition), with statistical significance determined by Mann-Whitney test. ( $\mathbf{D}$ and $\mathbf{E})$ Effect of E2 treatment on the amount of secreted MMP12 and MMP12 mRNA, respectively, in Mox macrophages ( $n=7$ per condition) with statistical significance determined by ANOVA. B-E show results from experiments that contained all conditions but are separated here to better emphasize distinct points; Mox samples lacking E2 in $\mathbf{B}$ and $\mathbf{C}$ are therefore the Mox samples without E2 in D and E. (F) Effect of E2 treatment on MMP12 mRNA levels in human SMCs stimulated with $20 \%$ FBS $(n=4)$. The arrow indicates the MMP12 mRNA level in the serum-starved cells before FBS treatment. Statistical significance was determined by ANOVA. Graphs show box-and-whisker plots with Tukey's whiskers; the horizontal lines of boxes represent the 25 th percentile, the median, and the $75 \%$ percentile.

We have used the sexual dimorphism of the LDLR-null mouse, isolated mouse and human macrophages and SMCs, and genome-wide analysis to explore the basis of atheroprotection in females. Our results point to an important role for MMP12 and lead us to posit that an estrogen-dependent inhibition of macrophage MMP12 expression contributes to the protective effect of female sex on arterial stiffness and atherosclerotic lesion burden. The potential biomedical importance of our observations is emphasized by our finding that loss of MMP12 activity extends atheroprotection to males even in the presence of high cholesterol. 
A
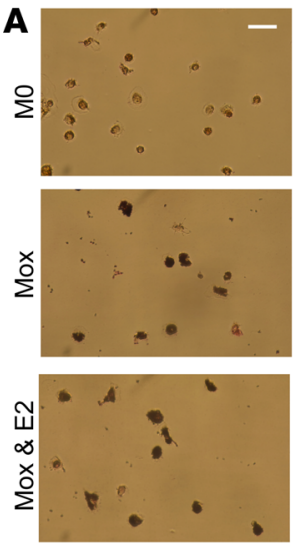

B

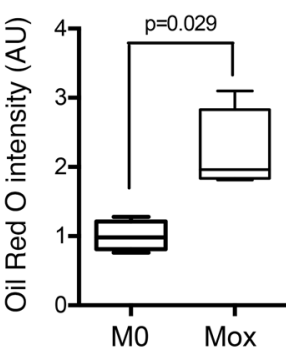

C

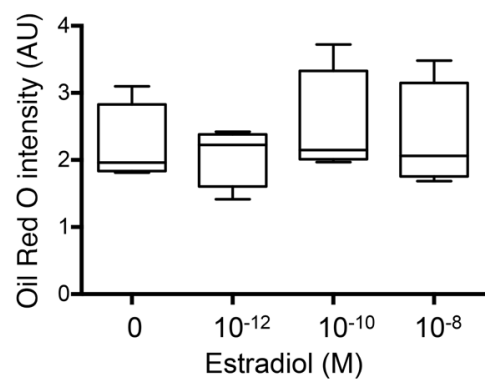

D

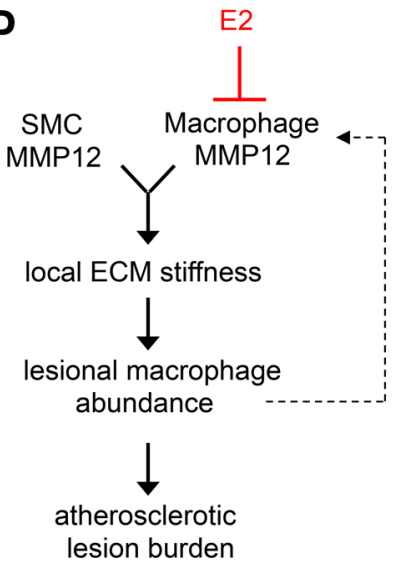

Figure 5. Oxidized LDL but not estrogen regulates lipid abundance in macrophages. (A) Female human PBDMs were differentiated into MO macrophages, treated with oxLDL, and exposed to selected concentrations of estradiol (E2) as in Figure 4. (A) Representative images of Oil Red 0 staining obtained at the end of the incubation with E2. Scale bar: $100 \mu \mathrm{m}$. (B and C) Quantification of Oil Red 0 staining from all samples; $n=4$ per condition. Statistical significance for B was determined using a Mann-Whitney test and for $\mathbf{C}$ was determined using ANOVA. The graphs show box and whisker plots with Tukey's whiskers; the horizontal lines of boxes represent the 25th percentile, the median, and the $75 \%$ percentile. (D) Summary model of estrogen-mediated protection from arterial stiffening and atherosclerosis.

\section{Methods}

Animals. C57BL/6 (WT), MMP12-null, and LDLR-null mice lines were obtained from Jackson Laboratories. Mice on the LDLR-null background were fed a diet containing $21 \%$ fat and $0.15 \%$ cholesterol (PMI LabDiet 40097) from 8 to 24 weeks. Aortas were isolated from the heart to the diaphragm, and a small portion of thoracic aortas near the diaphragm was used to determine the elastic modulus by AFM (see Supplemental Methods). The remainders of the aortas were used for en face Oil Red O staining. Aortic root cross-sections were obtained from fresh-frozen optimal cutting temperature medium-embedded hearts and immunostained with CD68, $\alpha$-SMA, or MMP12. See Supplemental Methods for details of Oil Red O staining, immunostaining, and use of aortas for isolation of RNA.

Human macrophage culture and treatment with estradiol. Peripheral blood monocytes from healthy donors (females aged 23-29 years) were obtained from the Human Immunology Core (University of Pennsylvania). The isolated monocytes from each donor were cultured at $10^{6}$ cells $/ \mathrm{ml}$ in complete media (RPMI-1640 media, 10\% human serum [Sigma-Aldrich], 1\% penicillin/streptomycin) and 20 $\mathrm{ng} / \mathrm{ml}$ human recombinant MCSF (r-MCSF) (PeproTech). For each donor, $10^{6}$ cells were seeded in ultralow attachment 24-well plates (Corning) with 3 to 4 replicates seeded per condition and differentiated independently as outlined below in each of the 2 independent experiments. The medium was refreshed after 3 days. On the fifth day, the medium was replaced with fresh r-MCSF-supplemented complete media with oxLDL (50 $\mu \mathrm{g} / \mathrm{ml}$; Alfa Aesar) and incubated for 1 day to differentiate the cells into Mox. On the sixth day, the medium was refreshed with r-MCSF-supplemented complete media in the absence (control) or presence of estradiol (E2) (Sigma-Aldrich), which was dissolved in ethanol at $10^{-5} \mathrm{M}$ and used at final concentrations of $10^{-12}, 10^{-10}$, and $10^{-8} \mathrm{M}$. The cells were incubated with E2 
for 48 hours. M0 cells were cultured in parallel throughout the incubations and refreshed at the times described above with culture medium lacking oxLDL and E2. On the eighth day, conditioned media was briefly centrifuged ( 7 minutes at $400 \times g$ at room temperature), and the remaining cells were collected after gentle scraping. A small portion of the cells was used to determine viability by exclusion of Trypan Blue Solution, 0.4\% (Thermo Fisher Scientific), using a Countess automated cell counter (Invitrogen), and the remainder were lysed in RNAqueous lysis buffer (Thermo Fisher Scientific). Both the conditioned media and cells lysates were stored at $-80^{\circ} \mathrm{C}$. MMP12 secretion was measured by ELISA (Abnova) according to the manufacturer's instructions using $100 \mu 1$ of the clarified conditioned media. Isolated RNA was analyzed by reverse transcription qualitative PCR (see Supplemental Methods).

To test for potential effects of E2 on lipid accumulation, replicate cultures were washed, fixed in $3.7 \%$ formaldehyde, incubated with $60 \%$ isopropanol for 5 minutes, stained with filtered Oil Red O $(0.4 \%$ in water; 12 minutes), and rinsed twice with $60 \%$ isopropanol and 3 times with PBS before imaging. Images of Oil Red O-stained macrophages were captured digitally and converted to 8-bit, inverted images in Fiji. Individual cells (typically $>30$ per independent replicate) were circled using the freehand tool; the area and integrated density were then determined using the Measure command. Background integrated density/area was similarly determined from several fields lacking cells for each sample, and the background signal was subtracted to yield net integrated density/area. The results were plotted as Oil Red O intensity.

Human SMC culture and treatment with estradiol. Female human aortic SMCs (Lonza) were cultured in SMC basal medium supplemented with SmGM-2 SingleQuots Supplements and Growth Factors (Lonza). Nearly confluent cells in 60-mm dishes were serum-starved for 2 days in SMC basal medium and $1 \mathrm{mg} / \mathrm{ml}$ heat-inactivated fatty acid-free BSA, pretreated with $0-10^{-8} \mathrm{M}$ estradiol for 30 minutes, and stimulated with $20 \%$ FBS in the continued presence of estradiol for 24 hours.

Mouse ovariectomy and $17 \beta$-estradiol replacement. $\mathrm{LDLR}^{-1}$ mice were bilaterally ovariectomized at approximately 6 weeks of age largely described (68). Mice were allowed 2 weeks to recover from the ovariectomy surgery before implantation of subcutaneous slow-release 17 $\beta$-estradiol pellets (Innovative Research of America) with the test concentration ( $8 \mu \mathrm{g} /$ day) similar to that used by others $(37,69,70)$. Mice were fed the high-fat diet in the absence or presence of exogenous E2 from 8 to 24 weeks old.

Statistics. Multiple comparisons were analyzed using 1-way ANOVA followed by Tukey's or Dunnett's multiple comparison tests. Comparisons between 2 conditions used Mann-Whitney tests. A $P$ value of less than 0.05 was considered statistically significant. Statistical analysis was performed in Prism GraphPad.

Study approval. Animal experimentation in this study was approved by the Institutional Animal Care and Use Committees of the Wistar Institute and University of Pennsylvania.

\section{Author contributions}

SLL, AB, KLS, and RKA designed the studies. SLL, AB, EAH, YB, PC, and JM conducted experiments and acquired data. SLL, AB, YB, PC, KLS, and RKA analyzed data. SLL, AB, YB, EP, KLS, and RKA contributed to manuscript writing and editing.

\section{Acknowledgments}

This work was supported by NIH grants AG047373 (to RKA), HL130037 (to KLS), the National Science Foundation Science and Technology Center for Engineering MechanoBiology award CMMI-1548571 (to RKA), and the Drexel Neuroinflammation and Gender Research Program (to KLS). The authors acknowledge technical assistance from Jessica Eager. We thank the Biomechanics Core of the Institute for Translational Medicine and Therapeutics at the University of Pennsylvania for performing the second harmonic generation microscopy and the Penn Cardiovascular Physiology Core for performing the blood pressure analysis.

Address correspondence to: Richard K. Assoian, University of Pennsylvania, Biomedical Research Building, 421 Curie Blvd, Room 805, Philadelphia, Pennsylvania 19104, USA. Phone: 215.898.7157; Email: assoian@ pennmedicine.upenn.edu. Or to: Kara L. Spiller, Drexel University, Bossone Research Center, 3140 Market St, Room 712, Philadelphia, Pennsylvania 19104, USA. Phone: 215.895.2215; Email: spiller@drexel.edu.

YB's present address is: Department of Pathology and Anatomical Sciences, Jacobs School of Medicine and Biomedical Sciences, The State University of New York, Buffalo, New York, USA. 
1. Tabas I, García-Cardeña G, Owens GK. Recent insights into the cellular biology of atherosclerosis. J Cell Biol. 2015;209(1):13-22.

2. Tabas I, Bornfeldt KE. Macrophage phenotype and function in different stages of atherosclerosis. Circ Res. 2016;118(4):653-667.

3. Gimbrone MA, García-Cardeña G. Endothelial cell dysfunction and the pathobiology of atherosclerosis. Circ Res. 2016;118(4):620-636.

4. Hahn C, Schwartz MA. Mechanotransduction in vascular physiology and atherogenesis. Nat Rev Mol Cell Biol. 2009;10(1):53-62.

5. Davies PF, Civelek M, Fang Y, Fleming I. The atherosusceptible endothelium: endothelial phenotypes in complex haemodynamic shear stress regions in vivo. Cardiovasc Res. 2013;99(2):315-327.

6. Steppan J, Barodka V, Berkowitz DE, Nyhan D. Vascular stiffness and increased pulse pressure in the aging cardiovascular system. Cardiol Res Pract. 2011;2011:263585.

7. Wang X, Keith JC, Struthers AD, Feuerstein GZ. Assessment of arterial stiffness, a translational medicine biomarker system for evaluation of vascular risk. Cardiovasc Ther. 2008;26(3):214-223

8. Duprez DA, Cohn JN. Arterial stiffness as a risk factor for coronary atherosclerosis. Curr Atheroscler Rep. 2007;9(2):139-144.

9. Mitchell GF, et al. Arterial stiffness and cardiovascular events: the Framingham Heart Study. Circulation. 2010;121(4):505-511.

10. Kothapalli D, et al. Cardiovascular protection by ApoE and ApoE-HDL linked to suppression of ECM gene expression and arterial stiffening. Cell Rep. 2012;2(5):1259-1271.

11. Huynh J, et al. Age-related intimal stiffening enhances endothelial permeability and leukocyte transmigration. Sci Transl Med. 2011;3(112):112ra122.

12. Wagenseil JE, Mecham RP. Vascular extracellular matrix and arterial mechanics. Physiol Rev. 2009;89(3):957-989.

13. Gerrity RG, Cliff WJ. The aortic tunica media of the developing rat. I. Quantitative stereologic and biochemical analysis. Lab Invest. 1975;32(5):585-600.

14. Mithieux SM, Weiss AS. Elastin. Adv Protein Chem. 2005;70:437-461.

15. Davidson J. Elastin: structure and biology In: Uitto J, Perejda AJ, eds. Connective Tissue Disease: molecular pathology of the extracellular matrix. New York: Marcel Dekker, Inc.; 1987:29-54.

16. Roycik MD, Myers JS, Newcomer RG, Sang QX. Matrix metalloproteinase inhibition in atherosclerosis and stroke. Curr Mol Med. 2013;13(8):1299-1313.

17. Werb Z, Gordon S. Elastase secretion by stimulated macrophages. Characterization and regulation. J Exp Med. 1975;142(2):361-377.

18. Banda MJ, Werb Z. Mouse macrophage elastase. Purification and characterization as a metalloproteinase. Biochem J. 1981;193(2):589-605.

19. Liu SL, et al. Matrix metalloproteinase-12 is an essential mediator of acute and chronic arterial stiffening. Sci Rep. 2015;5:17189

20. Liang J, et al. Macrophage metalloelastase accelerates the progression of atherosclerosis in transgenic rabbits. Circulation. 2006;113(16):1993-2001.

21. Johnson JL, George SJ, Newby AC, Jackson CL. Divergent effects of matrix metalloproteinases 3, 7, 9, and 12 on atherosclerotic plaque stability in mouse brachiocephalic arteries. Proc Natl Acad Sci U S A. 2005;102(43):15575-15580.

22. Lijnen HR. Metalloproteinases in development and progression of vascular disease. Pathophysiol Haemost Thromb. 2003;33(5-6):275-281.

23. Johnson JL, et al. A selective matrix metalloproteinase-12 inhibitor retards atherosclerotic plaque development in apolipoprotein E-knockout mice. Arterioscler Thromb Vasc Biol. 2011;31(3):528-535.

24. Amor M, et al. Identification of matrix metalloproteinase-12 as a candidate molecule for prevention and treatment of cardiometabolic disease. Mol Med. 2016;22:487-496.

25. Traylor M, et al. A novel MMP12 locus is associated with large artery atherosclerotic stroke using a genome-wide age-at-onset informed approach. PLoS Genet. 2014;10(7):e1004469.

26. Mahdessian $\mathrm{H}$, et al. Integrative studies implicate matrix metalloproteinase-12 as a culprit gene for large-artery atherosclerotic stroke. J Intern Med. 2017;282(5):429-444.

27. Soler A, et al. Elevated 20-HETE in metabolic syndrome regulates arterial stiffness and systolic hypertension via MMP12 activation. J Mol Cell Cardiol. 2018;117:88-99.

28. Mathur P, Ostadal B, Romeo F, Mehta JL. Gender-related differences in atherosclerosis. Cardiovasc Drugs Ther. 2015;29(4):319-327.

29. Rosen SE, Henry S, Bond R, Pearte C, Mieres JH. Sex-specific disparities in risk factors for coronary heart disease. Curr Atheroscler Rep. 2015;17(8):49.

30. Kallen AN, Pal L. Cardiovascular disease and ovarian function. Curr Opin Obstet Gynecol. 2011;23(4):258-267.

31. Suzuki H, Kondo K. Pulse wave velocity in postmenopausal women. Pulse (Basel). 2013;1(1):4-13.

32. Tomiyama $\mathrm{H}$, et al. Influences of age and gender on results of noninvasive brachial-ankle pulse wave velocity measurement - a survey of 12517 subjects. Atherosclerosis. 2003;166(2):303-309.

33. Rajkumar C, et al. Hormonal therapy increases arterial compliance in postmenopausal women. J Am Coll Cardiol. 1997;30(2):350-356

34. Takahashi K, et al. Impact of menopause on the augmentation of arterial stiffness with aging. Gynecol Obstet Invest. 2005;60(3):162-166

35. Kleinert H, Wallerath T, Euchenhofer C, Ihrig-Biedert I, Li H, Förstermann U. Estrogens increase transcription of the human endothelial NO synthase gene: analysis of the transcription factors involved. Hypertension. 1998;31(2):582-588.

36. Meyer MR, et al. G protein-coupled estrogen receptor protects from atherosclerosis. Sci Rep. 2014;4:7564.

37. Egan KM, et al. COX-2-derived prostacyclin confers atheroprotection on female mice. Science. 2004;306(5703):1954-1957.

38. Tangirala RK, Rubin EM, Palinski W. Quantitation of atherosclerosis in murine models: correlation between lesions in the aortic origin and in the entire aorta, and differences in the extent of lesions between sexes in LDL receptor-deficient and apolipoprotein E-deficient mice. J Lipid Res. 1995;36(11):2320-2328.

39. Anea CB, et al. Matrix metalloproteinase 2 and 9 dysfunction underlie vascular stiffness in circadian clock mutant mice. Arterioscler Thromb Vasc Biol. 2010;30(12):2535-2543.

40. Newby AC. Matrix metalloproteinases regulate migration, proliferation, and death of vascular smooth muscle cells by degrading matrix and non-matrix substrates. Cardiovasc Res. 2006;69(3):614-624. 
41. Matsumoto S, et al. Expression and localization of matrix metalloproteinase-12 in the aorta of cholesterol-fed rabbits: relationship to lesion development. Am J Pathol. 1998;153(1):109-119.

42. Yamada S, et al. Matrix metalloproteinase 12 accelerates the initiation of atherosclerosis and stimulates the progression of fatty streaks to fibrous plaques in transgenic rabbits. Am J Pathol. 2008;172(5):1419-1429.

43. Wang JC, Bennett M. Aging and atherosclerosis: mechanisms, functional consequences, and potential therapeutics for cellular senescence. Circ Res. 2012;111(2):245-259.

44. Silva SD, Nonogaki S, Soares FA, Kowalski LP. p16 (INK4a) has clinicopathological and prognostic impact on oropharynx and larynx squamous cell carcinoma. Braz J Med Biol Res. 2012;45(12):1327-1333.

45. Melk A, Schmidt BM, Takeuchi O, Sawitzki B, Rayner DC, Halloran PF. Expression of p16INK4a and other cell cycle regulator and senescence associated genes in aging human kidney. Kidney Int. 2004;65(2):510-520.

46. Ueberham E, et al. Global increase of p16INK4a in APC-deficient mouse liver drives clonal growth of p16INK4a-negative tumors. Mol Cancer Res. 2015;13(2):239-249.

47. Niu H, et al. Matrix metalloproteinase 12 modulates high-fat-diet induced glomerular fibrogenesis and inflammation in a mouse model of obesity. Sci Rep. 2016;6:20171.

48. Moore KJ, Sheedy FJ, Fisher EA. Macrophages in atherosclerosis: a dynamic balance. Nat Rev Immunol. 2013;13(10):709-721.

49. Stoka KV, et al. Effects of Increased Arterial Stiffness on Atherosclerotic Plaque Amounts. J Biomech Eng. 2018;140(5).

50. Tham DM, et al. Angiotensin II injures the arterial wall causing increased aortic stiffening in apolipoprotein E-deficient mice. Am J Physiol Regul Integr Comp Physiol. 2002;283(6):R1442-R1449.

51. Daugherty A, Manning MW, Cassis LA. Angiotensin II promotes atherosclerotic lesions and aneurysms in apolipoprotein E-deficient mice. J Clin Invest. 2000;105(11):1605-1612.

52. Van Herck JL, et al. Impaired fibrillin-1 function promotes features of plaque instability in apolipoprotein E-deficient mice. Circulation. 2009;120(24):2478-2487.

53. Maedeker JA, et al. Hypertension and decreased aortic compliance due to reduced elastin amounts do not increase atheroscle rotic plaque accumulation in $\mathrm{Ldlr}^{-/-}$mice. Atherosclerosis. 2016;249:22-29.

54. Thomas AC, Sala-Newby GB, Ismail Y, Johnson JL, Pasterkamp G, Newby AC. Genomics of foam cells and nonfoamy macrophages from rabbits identifies arginase-I as a differential regulator of nitric oxide production. Arterioscler Thromb Vasc Biol. 2007;27(3):571-577.

55. Kohn JC, Lampi MC, Reinhart-King CA. Age-related vascular stiffening: causes and consequences. Front Genet. $2015 ; 6: 112$.

56. Chow MJ, Turcotte R, Lin CP, Zhang Y. Arterial extracellular matrix: a mechanobiological study of the contributions and interactions of elastin and collagen. Biophys J. 2014;106(12):2684-2692.

57. Bäck M, Ketelhuth DF, Agewall S. Matrix metalloproteinases in atherothrombosis. Prog Cardiovasc Dis. 2010;52(5):410-428.

58. Nagase H. Substrate Specificity of MMPs. In: Cancer Drug Discovery and Development. 2001:39-66. https://www.springer. com/us/book/9780896036680.

59. Wells JM, Gaggar A, Blalock JE. MMP generated matrikines. Matrix Biol. 2015;44-46:122-129.

60. Dean RA, Cox JH, Bellac CL, Doucet A, Starr AE, Overall CM. Macrophage-specific metalloelastase (MMP-12) truncates and inactivates ELR+ CXC chemokines and generates CCL2, -7, -8, and -13 antagonists: potential role of the macrophage in terminating polymorphonuclear leukocyte influx. Blood. 2008;112(8):3455-3464.

61. Shipley JM, Wesselschmidt RL, Kobayashi DK, Ley TJ, Shapiro SD. Metalloelastase is required for macrophage-mediated proteolysis and matrix invasion in mice. Proc Natl Acad Sci U S A. 1996;93(9):3942-3946.

62. Stawski L, Haines P, Fine A, Rudnicka L, Trojanowska M. MMP-12 deficiency attenuates angiotensin II-induced vascular injury, M2 macrophage accumulation, and skin and heart fibrosis. PLoS One. 2014;9(10):e109763.

63. Churg A, et al. Macrophage metalloelastase mediates acute cigarette smoke-induced inflammation via tumor necrosis factor-alpha release. Am J Respir Crit Care Med. 2003;167(8):1083-1089.

64. Nénan S, et al. Analysis of the inflammatory response induced by rhMMP-12 catalytic domain instilled in mouse airways. Int Immunopharmacol. 2005;5(3):511-524.

65. Lee JT, et al. Macrophage metalloelastase (MMP12) regulates adipose tissue expansion, insulin sensitivity, and expression of inducible nitric oxide synthase. Endocrinology. 2014;155(9):3409-3420.

66. Marchant DJ, et al. A new transcriptional role for matrix metalloproteinase-12 in antiviral immunity. Nat Med. 2014;20(5):493-502.

67. Lieb W, et al. Multimarker approach to evaluate correlates of vascular stiffness: the Framingham Heart Study. Circulation. 2009;119(1):37-43.

68. Ström JO, et al. Ovariectomy and 17ק-estradiol replacement in rats and mice: a visual demonstration. J Vis Exp. $2012 ; 64:$ e4013.

69. Bourassa PA, Milos PM, Gaynor BJ, Breslow JL, Aiello RJ. Estrogen reduces atherosclerotic lesion development in apolipoprotein E-deficient mice. Proc Natl Acad Sci U S A. 1996;93(19):10022-10027.

70. Hodgin JB, Krege JH, Reddick RL, Korach KS, Smithies O, Maeda N. Estrogen receptor $\alpha$ is a major mediator of $17 \beta$-estradiol's atheroprotective effects on lesion size in Apoe ${ }^{-/-}$mice. J Clin Invest. 2001;107(3):333-340.

71. Nakashima Y, Plump AS, Raines EW, Breslow JL, Ross R. ApoE-deficient mice develop lesions of all phases of atherosclerosis throughout the arterial tree. Arterioscler Thromb. 1994;14(1):133-140. 